\title{
EDITORIAL
}

\section{DE MARZO. HACIA EL FIN DE LA TUBERCULOSIS SIN DEJAR A NADIE ATRÁS}

María C. Vázquez Torres (1), Raquel González Rubio (2) y Julia del Amo Valero (3)

(1) Jefa de Área de Prevención y Coordinación Multisectorial y Autonómica. Plan Nacional sobre el Sida. Madrid. España.

(2) Técnica Superior Externa TRAGSATEC. Plan Nacional sobre el Sida. Madrid. España.

(3) Directora del Plan Nacional sobre el Sida. Madrid. España.

La tuberculosis (TB) persiste como una amenaza para la salud pública desde su declaración como tal en 1991, a pesar de los esfuerzos realizados para su control. Globalmente, la TB es la primera causa de muerte en el mundo debida a un único agente infeccioso. En 2018 se diagnosticaron 10 millones de casos nuevos, y alrededor de 1,5 millones de personas fallecieron por esta causa. Geográficamente, la distribución es desigual y la epidemia se concentra en determinadas regiones y países con elevada carga de enfermedad ${ }^{(1)}$.

Esta dolencia ha estado presente en los compromisos sanitarios de la comunidad internacional. La Organización Mundial de la Salud, como Agencia Especializada de las Naciones Unidas, aprobó la Estrategia "Poner fin a la tuberculosis" en su Asamblea Mundial, incluyendo como metas la reducción de la mortalidad en un $90 \%$ y la tasa de incidencia en un $80 \%$ para 2035 . Dicha estrategia tiene un marcado compromiso con las personas más vulnerables, que son las más afectadas por la epidemia ${ }^{(2)}$.

Asimismo, en el objetivo 3 de la Agenda 2030 por un Desarrollo Sostenible, relacionado con la salud y el bienestar de las personas, se incluye en su meta 3.3 la eliminación de la epidemia global de TB para $2030^{(3)}$. La lucha internacional contra la TB tuvo su impulso más reciente con la Reunión de Alto Nivel
(RAN) celebrada en septiembre de 2018 con ocasión de la Asamblea General de Naciones Unidas, y que con el lema "Unidos para poner fin a la TB: Una respuesta global urgente a una epidemia global" señala de forma inequívoca el refuerzo de las acciones necesarias a nivel global para alcanzar los objetivos acordados, así como los retos persistentes y nuevos que esta enfermedad está suponiendo para las personas y los sistemas sanitarios ${ }^{(4)}$.

Globalmente se observa un descenso de la tasa media de incidencia de un 1,6\% anual entre 2000 y 2018, y una reducción del 11\% en el número total de muertes por TB entre 2015 y $2018^{(1)}$. En los países de la Unión Europea/Área Económica Europea se confirmaron 37.700 casos de TB (datos de 2017), y un $7 \%$ de todos los casos con información disponible acerca de resistencias resultaron ser casos multirresistentes. De ellos, un 24,3\% que disponían de información sobre antibiograma de segunda línea resultaron ser casos XDR (extensivamente resistentes), y en ambos casos con rangos muy amplios sobre los que incidir y mejorar. Asimismo, se observó un descenso medio anual de entre el $2 \%$ y el $6 \%$ en todos los grupos de edad entre los años 2008 y $2017^{(5)}$.

En España, los datos para 2018 muestran una tasa estimada de incidencia de 9,43 casos por cada 100.000 habitantes, y de incidencia de TB pulmonar en torno a 6,78 casos 
por cada 100.000 , con un descenso acumulado en la incidencia estimada de TB entre 2015 y 2018 del 11,4\% y en la incidencia estimada de TB pulmonar del 13\%. No obstante, la tendencia es irregular en este corto periodo y plantea retos de consolidación y avance en el futuro más inmediato, los cuales nos han de alejar de la complacencia. Asimismo, la distribución de la enfermedad y de la incidencia estimada es muy desigual entre territorios y comunidades autónomas, y el descenso en la razón de tasas entre menores y mayores de 15 años progresa con mucha lentitud desde 2015.

En 2019, el Consejo Interterritorial del Servicio Nacional de Salud de España (CISNS) aprobó el Plan para la prevención y control de la TB en España (en adelante, el Plan TB), respondiendo a la demanda y necesidad profesional en materia de salud pública y en un escenario de concertación y consonancia con las estrategias y compromisos globales. El objetivo general es detener la transmisión gracias al acceso universal a prevención, diagnóstico y tratamiento, centrándose en la transmisión de las formas pulmonares y en las formas multi y extensivamente resistentes. Esto incluye objetivos y metas relativas a la reducción de la tasa de incidencia de TB global y pulmonar, la mejora del éxito de tratamiento y la mejora del conocimiento e información sobre la epidemia, con una vocación explícita de "no dejar a nadie atrás"(6).

EL Plan TB da continuidad a las líneas estratégicas en materia de diagnóstico (incidiendo en la reducción de la demora diagnóstica y la identificación de infección) y de vigilancia epidemiológica (pretendiendo una mayor exhaustividad y conocimiento de la distribución, la realización y finalización de los estudios de contactos y las acciones apropiadas de prevención y tratamiento, incluidas las acciones de coordinación nacional e internacional cuando se precisen). De modo particular, se incorpo- ra de manera integral la intersectorialidad y la transversalidad de las acciones en cada una de ellas $^{(6)}$. Controlar esta enfermedad, cuyos aliados históricos han sido la exclusión y la pobreza (y en décadas pasadas, la infección por el VIH), pasa de modo obligado por atender esta realidad. Para ello se coordinan e impulsan acciones intersectoriales con profesionales del ámbito de los servicios sociales y de migraciones (entre otros), además de iniciar una hoja de ruta para el acceso universal a los tratamientos para quienes lo precisen e impulsar la incorporación de la evidencia, de los productos e innovaciones terapéuticas y diagnósticas al arsenal disponible en el Sistema Nacional de Salud para la lucha contra esta enfermedad prevenible y curable. Especialmente, las más recientes recomendaciones para el tratamiento de las formas multirresistentes, obliga a los sistemas sanitarios a responder de modo acorde y oportuno ${ }^{(7)}$.

El Plan TB propone la conformación de equipos de expertos en las comunidades autónomas en sus respectivos ámbitos competenciales y, muy particularmente, para el abordaje de las formas resistentes en cualquiera de sus formas e intensidades y ya operativas en determinados territorios. Asimismo, anima a una propuesta de trabajo flexible a los modelos organizativos de los servicios asistenciales y de salud pública, y al intercambio, coordinación y generación de conocimiento de modo concertado. La reconocida y dilatada experiencia y el esfuerzo en nuestro país de los profesionales sanitarios del ámbito asistencial y de la salud pública (y de otros sectores) aporta un conocimiento y unas buenas prácticas de elevada calidad y valor añadido, generadas desde los programas implementados en las comunidades autónomas al servicio de los objetivos comunes.

La consecución de los objetivos y metas del Plan TB requiere su materialización $\mathrm{y}$ 
escenarios de aplicación de los esfuerzos necesarios reconocidos y de los compromisos adquiridos a nivel nacional e internacional.

Con motivo del Día Mundial contra la Tuberculosis (24 de Marzo), la RESP realizará un número monográfico centrado en esta infección y enfermedad, para lo cual invita a todos los profesionales implicados en su manejo y abordaje a remitir sus manuscritos para publicación antes del día 28 de febrero de 2020.

\section{BIBLIOGRAFÍA}

1. Global tuberculosis report 2019. Geneva: World Health Organization; 2019. Disponible en: https://apps.who.int/ iris/bitstream/handle/10665/329368/9789241565714-eng. pdf?ua $=1$.

2. The END TB Strategy. Geneva. World Health Organization; 2019. Disponible en: https://www.who.int/ tb/strategy/End_TB_Strategy.pdf.

3. Transformar nuestro mundo: laAgenda 2030 para el Desarrollo Sostenible Resolución A/70/L.1 . Disponible en: https://www. un.org/ga/search/view_doc.asp?symbol=A/70/L.1\&Lang=S.
4. United to end Tuberculosis: An urgent global response to a global epidemic. Political declaration on the High-Level Meeting of the United Nations General Assembly on the fight against TB. Disponible en: https://www.who.int/tb/ unhlmonTBDeclaration.pdf.

5. Tuberculosis situation in the EU/EEA, 2017. Findings from the joint report Tuberculosis surveillance and monitoring in Europe by ECDC and WHO Regional Office for Europe. Stockholm, 19 March 2019. Disponible en: https://www.ecdc.europa.eu/en/publications-data/presentation-tuberculosis-situation-eueea-2017.

6. Grupo de trabajo Plan Prevención y Control de la Tuberculosis. Plan para la prevención y control de la tuberculosis en España. Comisión de Salud Pública del Consejo Interterritorial del Sistema Nacional de Salud. Ministerio de Sanidad, Consumo y Bienestar Social, marzo 2019. disponible en: https://www.mscbs.gob.es/profesionales/saludPublica/prevPromocion/PlanTuberculosis/docs/PlanTB2019.pdf.

7. WHO consolidated guidelines on drug-resistant tuberculosis treatment. Geneva: World Health Organization; 2019. Licence: CC BY-NC-SA 3.0 IGO. Disponible en: https://apps.who.int/iris/bitstream/hand le/10665/311389/9789241550529-eng.pdf?ua=1. 